\title{
Exploring the QCD phase diagram through relativistic heavy ion collisions
}

\author{
Bedangadas Mohanty ${ }^{1, a}$ \\ ${ }^{1}$ School of Physical Sciences, National Institute of Science Education and Research, Bhubaneswar 751005, \\ India
}

\begin{abstract}
We present a review of the studies related to establishing the QCD phase diagram through high energy nucleus-nucleus collisions. We particularly focus on the experimental results related to the formation of a quark-gluon phase, crossover transition and search for a critical point in the QCD phase diagram.
\end{abstract}

\section{Introduction}

Physical systems can be made to undergo phase transitions by varying parameters such as the temperature $(T)$ or a chemical potential $(\mu)$ of the system. Systems whose underlying interactions are strong interactions, are not different. In the theory of strong interactions, Quantum Chromodynamics (QCD), there are distinct conserved quantities. For a grand canonical ensemble of strongly interacting particles, the conserved baryon, electric charge and strangeness numbers are associated with the corresponding chemical potentials $\mu_{B}, \mu_{Q}$, and $\mu_{S}$, respectively. So for a system with strong interactions one can lay out the phase diagram with axes being $T, \mu_{B}, \mu_{Q}$, and $\mu_{S}$. Experimentally such a system of strong interactions can be created by colliding two nuclei at high energy. However, in such a system one can only vary to an appreciable extent $T$ and $\mu_{B}$ (values of $\mu_{Q}$, and $\mu_{S}$ are small [1]). This can be done by varying the center of mass energies $\left(\sqrt{s_{\mathrm{NN}}}\right)$ of the collision of the two heavy nuclei $[2,3]$. Hence through relativistic heavy-ion collisions we can explore a two dimensional phase diagram, $T$ versus $\mu_{B}$, of strong interactions [4].

Such a phase diagram has several distinct phase structures. Some of which are: (a) high temperature and/or density phase of deconfined quarks and gluons (QGP), (b) low temperature and/or density phase of hadrons, (c) nature of quark-hadron transition is crossover for the small $\mu_{B}$ part of the phase diagram and first order for the rest (large $\mu_{B}$ ) of the phase diagram, and (d) end point of the first order phase transition line (called the critical point (CP)).

In this paper, we will present the experimental results that support the formation of a deconfined phase of quarks and gluons in high energy nuclear collisions, experimental and theoretical evidences towards establishing the quark-hadron transition as a crossover and progress towards the search for the critical point on the phase diagram.

\footnotetext{
ae-mail: bedanga@niser.ac.in
} 


\section{Quark-Gluon Phase}

The experimental programs at the Relativistic Heavy Ion Collider (RHIC) facility [5-7] and the Large Hadron Collider (LHC) facility [8] have conclusively provided evidences towards the formation of a deconfined state of matter in heavy-ion collisions where the relevant degrees of freedom are quarks and gluons. In this section we will only discuss a subset of these results. We will also show that these signatures get gradually turned-off when the beam energy is dialled down to low enough values of $\sqrt{s_{\mathrm{NN}}}$ (RHIC Beam Energy Scan (BES) Program $[9,10]$ ), where temperature and energy density achieved are possibly below those predicted by QCD calculations for a quark-hadron transition. Typical temperature and energy density for phase transition are of the order of $170 \mathrm{MeV}$ and $1 \mathrm{GeV} / \mathrm{fm}^{3}$, respectively [11].

\subsection{Nuclear Modification Factor}

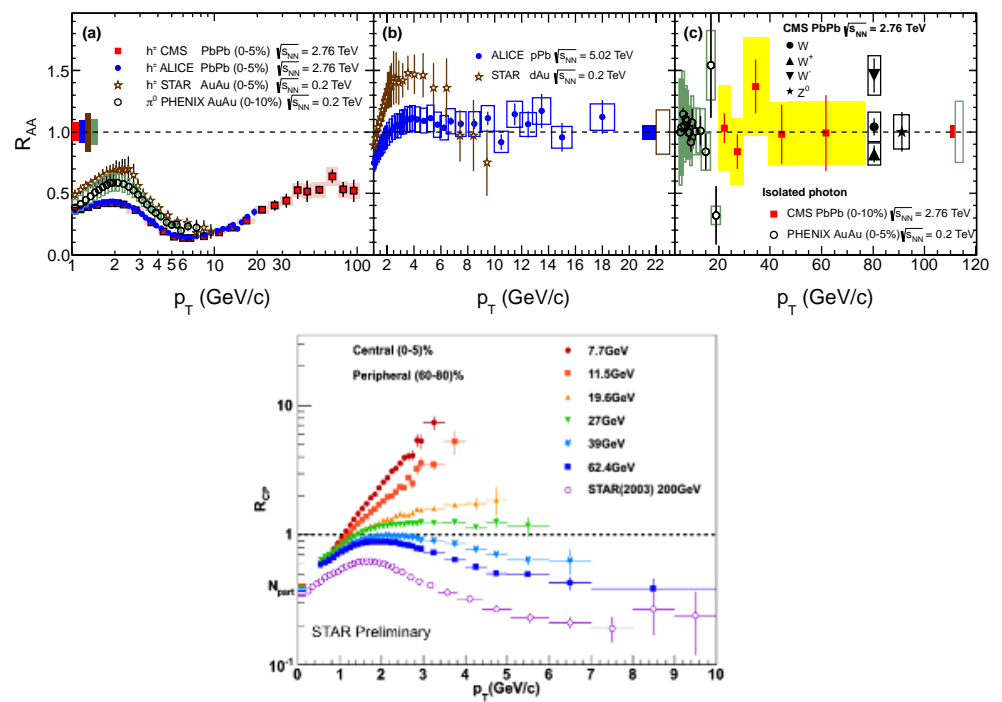

Figure 1. (Color online) Top panel: (a) Nuclear modification factor $R_{\mathrm{AA}}$ of charged hadrons measured by LHC experiments (ALICE [12] and CMS [13]) and $R_{\mathrm{AA}}$ of hadrons measured by RHIC experiments (charged hadrons by STAR [14] and $\pi^{0}$ by PHENIX [15]). (b) Comparison of nuclear modification factor for charged hadrons versus $p_{T}$ for minimum bias collisions in $d+\mathrm{Au}$ collisions at RHIC [14] and $p+\mathrm{Pb}$ collisions at LHC [16]. (c) The $R_{\mathrm{AA}}$ versus $p_{T}$ for isolated photons in central nucleus-nucleus collisions at RHIC [17] and LHC [18]. Also shown are the $R_{\mathrm{AA}}$ of $W^{ \pm}$[19] and $Z$ bosons [20] at LHC energies. The boxes around the data denote $p_{T^{-}}$ dependent systematic uncertainties. The systematic uncertainties on the normalisation are shown as boxes at $R_{\mathrm{AA}}$ $=1$. Bottom panel: Nuclear modification factor $\left(R_{\mathrm{CP}}\right)$ versus transverse momentum for charged hadrons in RHIC BES program [21].

The nuclear modification factor $\left(R_{\mathrm{AA}}\right)$ is defined as $\frac{d N_{A A} / d \eta d^{2} p_{T}}{T_{A B} d \sigma_{N N} / d \eta d^{2} p_{T}}$, where $T_{A B}=N_{\text {binary }} / \sigma_{\text {inelastic }}^{p p}$ is the overlap integral (with $N_{\text {binary }}$ being the number of binary collisions commonly estimated from Glauber model calculation) and $d \sigma_{N N} / d \eta d^{2} p_{T}$ is the cross section of charged hadron production in $p+p$ collisions. $R_{\mathrm{AA}}$ at high transverse momentum $\left(p_{T}\right)$ having a value of less than one is attributed to energy loss of partons in QGP and this phenomenon is referred to as the jet quenching in a dense partonic matter [22]. 

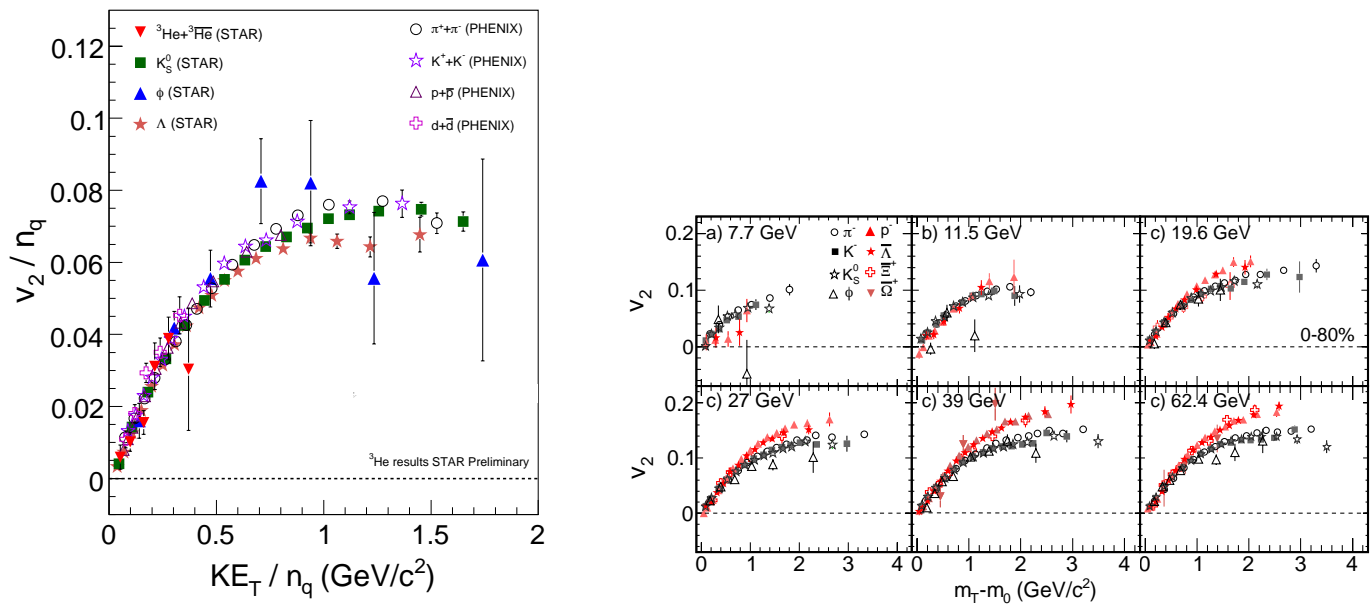

Figure 2. (Color online) Left panel: Elliptic flow $\left(v_{2}\right)$ divided by $n_{q}$ versus $\left(m_{T}-m\right) / n_{q}$ (denoted as $\left.K E_{T} / n_{q}\right)$ for various produced particles in $\mathrm{Au}+\mathrm{Au}$ collisions at $\sqrt{s_{\mathrm{NN}}}=200 \mathrm{GeV}$ [23]. Right panel: $v_{2}$ versus $m_{T}-m_{0}$ for various produced particles in RHIC BES program [24].

Figure 1 shows the $R_{\mathrm{AA}}$ of various particles produced in nucleus-nucleus collisions at RHIC and LHC. In Fig. 1(a), we observe that it is $R_{\mathrm{AA}}<1$ both at RHIC and LHC. In Fig. 1(b), we observe that the nuclear modification factors for $d+\mathrm{Au}$ collisions at $\sqrt{s_{\mathrm{NN}}}=200 \mathrm{GeV}$ [14] and $p+\mathrm{Pb}$ collisions at $\sqrt{s_{\mathrm{NN}}}=5.02 \mathrm{TeV}$ [16] are greater than unity for $p_{T}>2 \mathrm{GeV} / c$ both at RHIC and LHC. We do not expect formation of a dense partonic matter in such collisions. The nuclear modification factor value in $p(d)+\mathrm{A}$ collisions being close to unity suggests that $p(d)+\mathrm{A}$ collisions can be considered as due to superposition of several $p+p$ collisions. It acts as an experimental support to the view that a hot and dense medium of color charges is formed in nucleus-nucleus collisions at RHIC and LHC. In Fig. 1(c), we show the $R_{\mathrm{AA}}$ of particles that do not participate in strong interactions. These particles (photon [17, 18], $W^{ \pm}[19]$ and $Z$ [20] bosons) have a $R_{\mathrm{AA}} \sim 1$, indicating that the $R_{\mathrm{AA}}<1$, observed for hadrons in nucleus-nucleus collisions, are due to the strong interactions in a dense medium consisting of color charges. Going down in $\sqrt{s_{\mathrm{NN}}}$ gradually increases the value of $R_{\mathrm{CP}}$ (ratio of yields of charged particles in central to peripheral collisions normalized to respective $N_{\text {binary }}$ ) at high $p_{\mathrm{T}}$ towards unity and eventually becomes larger than unity for $p_{\mathrm{T}}>2 \mathrm{GeV} / c$ for $\sqrt{s_{\mathrm{NN}}}=11.5$ and $7.7 \mathrm{GeV}$ [21]. This indicates a turn-off of QGP signature at lower beam energies, although $p+\mathrm{A}$ collisions at these energies are required to quantitatively confirm the findings.

\subsection{Partonic Collectivity}

The elliptic flow $\left(v_{2}\right)$ is calculated as $\left\langle\cos 2\left(\phi-\Psi_{2}\right)\right\rangle$, where $\phi$ denotes the azimuthal angle of the produced particles and $\Psi_{2}$ denotes the orientation of the second order event plane (plane subtended by the impact parameter and beam axis). A new observation was made at RHIC in the measurement of $v_{2}$ for various hadrons. For $p_{\mathrm{T}}>2 \mathrm{GeV} / c$, there as a clear splitting of $v_{2}$ observed on the basis of baryons and mesons. All the baryons were found to have similar $v_{2}$ and in turn all the mesons were to found to have similar $v_{2}$ which was lower compared to the baryons. Dividing the $v_{2}$ by the number of constituent quarks $\left(n_{q}=2\right.$ for mesons and 3 for baryons) and plotting it versus $\left(m_{T}-m_{0}\right) / n_{q}$ (where $m_{T}$ is the 


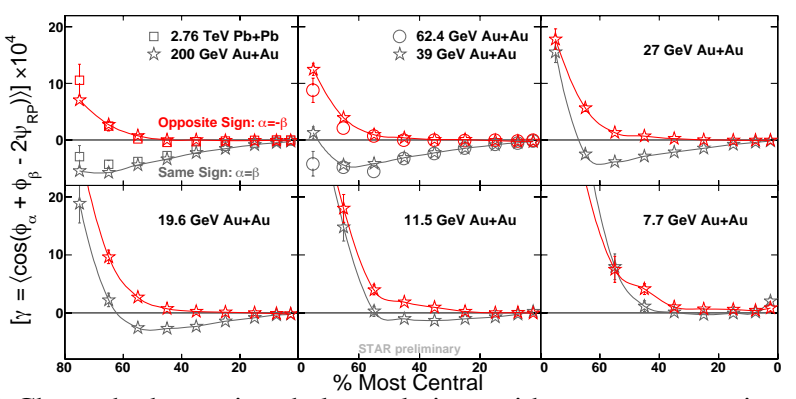

Figure 3. (Color online) Charge hadron azimuthal correlations with respect to reaction plane angle as a function of centrality for nucleus-nucleus collisions at midrapidity[27].

transverse mass and $m_{0}$ is the rest mass of the hadron) showed a remarkable scaling for most of the measured hadrons and light nuclei (shown in Fig. 2 left panel) [23]. All these particles have different interaction cross sections, freeze-out at different times and have large variations in their masses, yet they show a similar $v_{2} / n_{q}$ when plotted versus $\left(m_{T}-m_{0}\right) / n_{q}$. This indicates that a substantial amount of the measured $v_{2}$ is developed in the partonic phase and the contribution from hadronic phase is small $[25,26]$. Hence these measurements support the idea of formation of a medium with quarks and gluons. Fig. 2 (right panel) shows that the baryon-meson splitting (for $m_{T}-m_{0}>1 \mathrm{GeV} / c^{2}$ and source of this partonic collectivity) reduces gradually as the beam energy is decreased and vanishes for $\sqrt{s_{\mathrm{NN}}}=11.5$ and $7.7 \mathrm{GeV}$ [24]. This indicates a turn-off of this QGP signature at lower beam energies, where the collision process perhaps does not create a matter of sufficiently high temperature and density.

\subsection{Dynamical Charge Correlations}

Figure 3 shows the results on charged hadron azimuthal correlations based on 3-particle correlation technique[28]. The results are from nucleus-nucleus collisions at $\sqrt{s_{\mathrm{NN}}}=7.7,11.5,19.6,27,39$, $64.2,200 \mathrm{GeV}[27,28]$ and $2.76 \mathrm{TeV}$ [29] at midrapidity between same-charge and opposite-charge hadrons with respect to the reaction plane angle $(\Psi)$. The observable, $\left\langle\cos \left(\phi_{\alpha}+\phi_{\beta}-2 \psi\right)\right\rangle$ represents the difference between azimuthal correlations (between two particles $\alpha$ and $\beta$ ) projected onto the direction of the angular momentum vector and correlations projected onto the collision event plane. The difference between the same charge and opposite charge correlations at the higher energies seems to be consistent with the predictions for existence of metastable domains in QCD vacuum leading to local parity violation. This phenomena needs deconfinement and chiral phase transitions which are expected to be achieved in heavy-ion collisions (also referred to as the Chiral Magnetic Effect (CME)) [30, 31].

We also observe that the difference in correlations between same and opposite charges seems to decrease as beam energy decreases and almost vanishes at $7.7 \mathrm{GeV}$. If the differences can be attributed to QCD transitions, absence of it may indicate absence of such transitions at the lower energies. The observable presented is parity-even, making it also susceptible to physical processes not related to CME. 

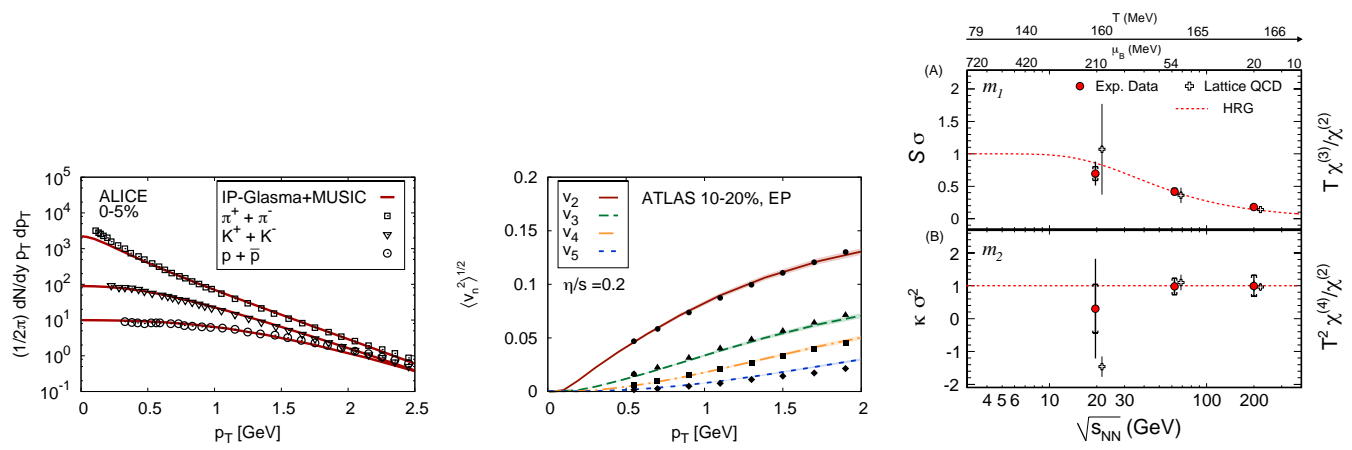

Figure 4. (Color online) Left panel: Hydrodynamical calculation of identified hadron transverse momentum spectra compared to experimental data from the ALICE collaboration, Middle panel: Root-mean-square anisotropic flow co-efficients as a function of transverse momentum compared to experimental data by the ATLAS collaboration [32], and Right panel: Comparison of lattice QCD and experimental data at RHIC. Experimentally measured ratios of cumulants of net-proton distributions, $S \sigma$ and $\kappa \sigma^{2}$, where $\sigma, S$ and $\kappa$ are the second, third and fourth moments, are shown as a function of $\sqrt{s_{\mathrm{NN}}}$ for impact parameter values of less than $3 \mathrm{fm}$ for $\mathrm{Au}+\mathrm{Au}$ collisions at RHIC [33]. Also plotted on the top scale are the chemical freeze-out values of $\mu_{B}$ and $T$ corresponding to $\sqrt{s_{\mathrm{NN}}}$ as obtained from a hadron resonance gas model, which assumes the system to be in chemical and thermal equilibrium at freeze-out $[2,3]$.

\section{Order of Phase Transition}

First principle QCD calculations on lattice at high temperature and $\mu_{B}=0 \mathrm{MeV}$ have established the quark-hadron transition to be a crossover [34]. The lattice chiral susceptibility $\chi\left(N_{s}, N_{t}\right)=$ $\partial^{2} /\left(\partial m_{u d}^{2}\right)(T / V) \cdot \log Z$, as a function of temperature was found to be independent of volume of the system. $m_{u d}$ is the mass of the light u,d quarks, $N_{s}$ is the spatial extension, $N_{\tau}$ the euclidean time extension, and $V$ the system volume.

Using this crossover equation of state for the quark-hadron transition in a hydrodynamic based model, the experimental data on invariant yields of charged hadrons and various order azimuthal anisotropy as a function of transverse momentum at LHC are nicely explained (shown in Fig 4) [32]. Lending indirect support to the transition being a crossover at small $\mu_{B}$. In addition, the ratio of baryon susceptibilities $\left(\chi^{n}\right)$ of various orders $(n)$ computed in lattice QCD calculations with a crossover at $\mu_{B}=0 \mathrm{MeV}$, when compared to experimental measurements of similar quantities using correlations between measured protons and anti-protons (reflected by the product of moments of net-proton distribution) also shows a remarkable agreement within errors.

The crossover temperature is obtained by looking for the point of sharpest change in temperature dependence of the chiral susceptibility $\left(\chi_{\bar{\psi} \psi}\right)$, the strange quark number susceptibility $\left(\chi_{s}\right)$ and the renormalized Polyakov-loop $(L)$ in the lattice calculations. Various lattice QCD estimates of chiral crossover temperature using $\chi_{\bar{\psi} \psi}$ have converged to a value of around $154 \mathrm{MeV}[35,36]$. However, the observables $\left(\chi_{s}\right.$ and $\left.L\right)$ that provide important insights into deconfining aspect of the crossover show a slightly higher crossover temperature of around $170 \mathrm{MeV}$. But with a width of around $15 \mathrm{MeV}$ in temperature estimates, it is difficult to make a concerte statement on the difference between various measures of the crossover temperatures. Moreover there are unresolved discussions on the establishment of Polyakov loop expectation and strange quark number susceptibilities to critical behaviour in the light quark mass regime [36]. 

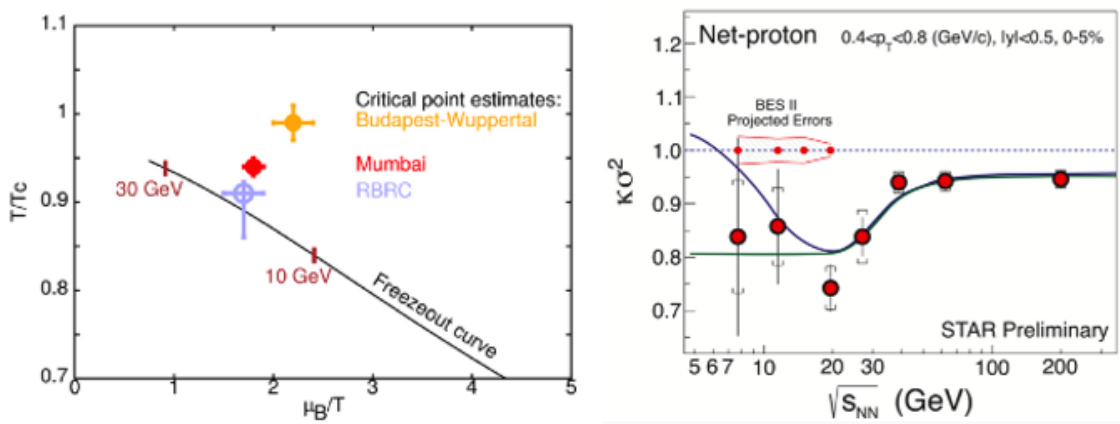

Figure 5. (Color online) Left panel: Estimates of position of critical point from various lattice QCD calculations [42, 43]. Right panel: $\kappa \sigma^{2}$ for net-proton distributions as a function of $\sqrt{s_{\mathrm{NN}}}$ in RHIC BES program [44]. Also shown are the projected statistical error in the second phase of BES program.

\section{Critical Point}

Several QCD based models predict the existence of an end point or critical point (CP) at high $\mu_{B}$ for the first order phase transition in the QCD phase diagram. However the exact location depends on the model assumptions used [37]. Given the ambiguity in predictions of CP in models, studies on lattice were expected to provide reliable estimates [38]. However lattice calculations at finite $\mu_{B}$ are difficult due to sign problem. There are several ways suggested to overcome this issue. (i) Reweighting the partition function in the vicinity of transition temperature and $\mu=0$ [39], (ii) Taylor expansion of thermodynamic observables in $\mu / \mathrm{T}$ about $\mu=0$ [40] and (iii) Choosing the chemical potential to be imaginary will make the fermionic determinant positive [41]. The first two methodologies yield an existence of $\mathrm{CP}$, whereas the third procedure gives a $\mathrm{CP}$ only when the first coefficient in the Taylor expansion of generic quark mass on the chiral critical surface $\left(m_{c}\right)$ as a function of $\mu / \mathrm{T}$ $\left(\frac{m_{c}(\mu)}{m_{c}(0)}=1+\sum_{k=1} c_{k}\left(\frac{\mu}{\pi T_{c}}\right)^{2 k}\right)$ is positive. The lattice calculations which yield a CP on phase diagram are shown in Fig. 5 [42, 43]. However these calculations still have to overcome some of the lattice artefacts like lattice spacing, physical quark masses, volume effect and continuum limit extrapolation.

In the experimental side, the characteristic signature of $\mathrm{CP}$ is large fluctuations in event-by-event conserved quantities like net-charge, net-baryon number and net-strangeness. The variance of these distributions $\left(\left\langle(\delta N)^{2}\right\rangle\right)$ are proportional to square of the correlation length $(\xi)$. It has been shown that higher moments $\left(\left\langle(\delta N)^{3}\right\rangle \sim \xi^{4.5}\right.$ and $\left.\left\langle(\delta N)^{4}\right\rangle \sim \xi^{7}\right)$ have stronger dependence on $\xi$ compared to variance and hence have higher sensitivity [45-47]. In addition the moments are related to susceptibilities [48]. Motivated by all these, experiments are studying the variable $\kappa \sigma^{2}$ of net-proton distributions (a proxy for net-baryon, see caption of Fig. 5), to search for the CP. $\kappa \sigma^{2}$ will be constant as per the Central limit theorem and hadron resonance gas model [49]. They have monotonic dependence with $\sqrt{s_{\mathrm{NN}}}$ for non-CP scenarios [50]. However as it is related to the ratio of baryon number susceptibilities in QCD models: $\kappa \sigma^{2}=\frac{\chi_{\mathrm{B}}^{(4)}}{\chi_{\mathrm{B}}^{(2)} / T^{2}}$ [51], close to $\mathrm{CP}$ it is expected to show a non-monotonic dependence on $\sqrt{s_{\mathrm{NN}}}$. Preliminary experimental results on $\kappa \sigma^{2}$ value for net-proton distributions measured in RHIC BES program are shown in the right panel of Fig. 5 [44]. Interesting trends are observed indicating that the $\mathrm{CP}$ if exists in the phase diagram, have to be below $\sqrt{s_{\mathrm{NN}}}=39 \mathrm{GeV}$ [52]. 


\section{Summary}

Relativistic heavy-ion collision experiments have seen distinct signatures which suggest that the relevant degrees of freedom at top RHIC and LHC energies in the initial stages of the collisions are quark and gluons and the system quickly approaches thermalization. The underlying mechanism for the fast thermalization is currently under study. Lowering the beam energies to $11.5 \mathrm{GeV}$ and below leads to a smooth turning-off of the QGP signatures, indicating that hadronic interactions dominate. These observations in turn further support the formation of partonic matter at higher energy collisions. Three such QGP signatures related to phenomena of jet-quenching, partonic collectivity and charge correlations (or chiral magnetic effect) are discussed in this paper.

The QCD calculations on lattice tell us that the above observed quark-hadron transition at LHC and top RHIC energies (small values of $\mu_{B} \sim 0 \mathrm{MeV}$ ) is a crossover. The theoretical evidence lies in the chiral susceptibility versus temperature not changing with change in volume. The chiral crossover temperature is found to be around $154 \mathrm{MeV}$. Other observables of quark-hadron crossover give a slightly higher values of the crossover temperature with large uncertainties. Using the lattice QCD based crossover equation of state in hydrodynamic models, one could explain the various measurements at RHIC and LHC. Two such measurements related to the transverse momentum distribution and azimuthal distribution of the produced particles in nucleus-nucleus collisions are presented in this paper. These lend indirect support from experimental measurements that the observed quark-hadron transition at LHC and top RHIC energies is a crossover.

Most QCD calculations on lattice continue to indicate the possible existence of critical point for $\mu_{B}>160 \mathrm{MeV}$, this possibility has not been ruled out from the data at RHIC. The exact location is not yet known unambiguously. The experimental measurements though encouraging are inconclusive. High event statistics measurements in the second phase of RHIC beam energy scan program should be able to provide a more quantitative and hence conclusive picture from the experimental side. Computing intensive lattice QCD calculations removing the remaining artefacts pertaining to lattice spacing, quark masses, system volume and choice of action will clear the picture from the theoretical side.

The progress towards establishing the phase diagram of strong interactions, one of the four basic interactions that occur in nature through relativistic heavy-ion collisions is significant. The phase structures of quark-gluon and hadronic phase has been distinctly identified, the transition is a crossover, with crossover temperatures varying between 150 - $175 \mathrm{MeV}$ (depending on the observable used), and both experiment and theory have ruled out the existence of the critical point for $\mu_{B}<$ $160 \mathrm{MeV}$ in the QCD phase diagram.

Acknowledgement: Financial support for the work is obtained from the DST Swarna Jayanti Fellowship.

\section{References}

[1] B. I. Abelev et al. [STAR Collaboration], Phys. Rev. C 79, 034909 (2009).

[2] J. Cleymans, H. Oeschler, K. Redlich and S. Wheaton, Phys. Rev. C 73, 034905 (2006).

[3] P. Braun-Munzinger and J. Stachel, Nature 448, 302 (2007).

[4] K. Rajagopal and F. Wilczek, In *Shifman, M. (ed.): At the frontier of particle physics, vol. $3 *$ 2061-2151 [hep-ph/0011333].

[5] J. Adams et al. [STAR Collaboration], ns," Nucl. Phys. A 757, 102 (2005).

[6] K. Adcox et al. [PHENIX Collaboration], Nucl. Phys. A 757, 184 (2005).

[7] M. Gyulassy and L. McLerran, Nucl. Phys. A 750, 30 (2005).

[8] R. Singh, L. Kumar, P. K. Netrakanti and B. Mohanty, arXiv:1304.2969 [nucl-ex]. 
[9] B. I. Abelev et al. [STAR Collaboration], Phys. Rev. C 81, 024911 (2010).

[10] B. Mohanty [STAR Collaboration], J. Phys. G 38, 124023 (2011).

[11] F. Karsch, Prog. Theor. Phys. Suppl. 153, 106 (2004) [hep-lat/0401031].

[12] K. Aamodt et al. [ALICE Collaboration], Phys. Lett. B 696, 30 (2011).

[13] S. Chatrchyan et al. [CMS Collaboration], Eur. Phys. J. C 72, 1945 (2012).

[14] J. Adams et al. [STAR Collaboration], Phys. Rev. Lett. 91, 072304 (2003).

[15] S. S. Adler et al. [PHENIX Collaboration], Phys. Rev. Lett. 96, 202301 (2006).

[16] B. Abelev et al. [ALICE Collaboration], arXiv:1210.4520 [nucl-ex].

[17] S. S. Adler et al. [PHENIX Collaboration], Phys. Rev. Lett. 94, 232301 (2005).

[18] S. Chatrchyan et al. [CMS Collaboration], Phys. Lett. B 710, 256 (2012).

[19] S. Chatrchyan et al. [CMS Collaboration], Phys. Lett. B 715, 66 (2012).

[20] S. Chatrchyan et al. [CMS Collaboration], Phys. Rev. Lett. 106, 212301 (2011).

[21] R. Horvat, A. Ilakovac, J. Trampetic and J. You, arXiv:1306.1239 [hep-th].

[22] X. -N. Wang and M. Gyulassy, Phys. Rev. Lett. 68, 1480 (1992).

[23] B. Mohanty, New J. Phys. 13, 065031 (2011).

[24] L. Adamczyk et al. [STAR Collaboration], arXiv:1301.2348.

[25] B. I. Abelev et al. [STAR Collaboration], Phys. Rev. Lett. 99, 112301 (2007).

[26] S. Afanasiev et al. [PHENIX Collaboration], Phys. Rev. Lett. 99, 052301 (2007).

[27] G. Wang [STAR Collaboration], Nucl. Phys. A904-905 2013 (2013) 248c.

[28] B. I. Abelev et al. [STAR Collaboration], Phys. Rev. Lett. 103 (2009) 251601.

[29] B. Abelev et al. [ALICE Collaboration], Phys. Rev. Lett. 110 (2013) 012301.

[30] K. Fukushima, D. E. Kharzeev and H. J. Warringa, Phys. Rev. D 78 (2008) 074033.

[31] D. Kharzeev, Phys. Lett. B 633 (2006) 260.

[32] C. Gale et al., Phys. Rev. Lett. 110, 012302 (2013).

[33] S. Gupta, X. Luo, B. Mohanty, H. G. Ritter and N. Xu, Science 332, 1525 (2011).

[34] Y. Aoki et al., Nature 443, 675 (2006).

[35] Y. Aoki et al., Nucl. Phys. A 830, 805C (2009).

[36] A. Bazavov et al., Phys. Rev. D 85, 054503 (2012).

[37] M. A. Stephanov, Prog. Theor. Phys. Suppl. 153, 139 (2004).

[38] R. V. Gavai and S. Gupta, Phys. Rev. D 71, 114014 (2005).

[39] Z. Fodor and S. D. Katz, JHEP 0404, 050 (2004).

[40] R. V. Gavai and S. Gupta, Phys. Rev. D 68, 034506 (2003).

[41] O. Philipsen, Nucl. Phys. A 830, 713C (2009).

[42] S. Gupta, Pramana 76, 801 (2011).

[43] S. Gupta, Prog. Theor. Phys. Suppl. 193, 11 (2012).

[44] X. Luo [ for the STAR Collaboration], arXiv:1306.3106 [nucl-ex].

[45] M. A. Stephanov, Phys. Rev. Lett. 102, 032301 (2009).

[46] M. A. Stephanov, Phys. Rev. Lett. 107, 052301 (2011).

[47] M. Asakawa, S. Ejiri and M. Kitazawa, Phys. Rev. Lett. 103, 262301 (2009).

[48] M. Cheng et al., Phys. Rev. D 79, 074505 (2009).

[49] P. Garg et al., Phys. Lett. B 726, 691 (2013).

[50] X. Luo, J. Xu, B. Mohanty and N. Xu, J. Phys. G 40, 105104 (2013).

[51] R. V. Gavai and S. Gupta, Phys. Lett. B 696, 459 (2011).

[52] M. M. Aggarwal et al. [STAR Collaboration], Phys. Rev. Lett. 105 (2010) 022302. 